\title{
Unifying decoherence and the Heisenberg Principle
}

\author{
Bas Janssens 1
}

Received: 28 April 2016 / Revised: 16 February 2017 / Accepted: 22 February 2017 /

Published online: 14 March 2017

(C) The Author(s) 2017. This article is published with open access at Springerlink.com

\begin{abstract}
We exhibit three inequalities involving quantum measurement, all of which are sharp and state independent. The first inequality bounds the performance of joint measurement. The second quantifies the trade-off between the measurement quality and the disturbance caused on the measured system. Finally, the third inequality provides a sharp lower bound on the amount of decoherence in terms of the measurement quality. This gives a unified description of both the Heisenberg uncertainty principle and the collapse of the wave function.
\end{abstract}

Keywords Decoherence · CP-maps · Uncertainty relations · Quantum measurement

Mathematics Subject Classification $81 \mathrm{P} 40$

\section{Introduction}

Initial Remark Modulo minor modifications, this manuscript has been on the ArXiv since 2006, under the identifier quant-ph/0606093. Since that time, several results have found their way into the literature [17-20,24,25]. This convinced me to submit the manuscript for publication.

In quantum mechanics, observables are modelled by self-adjoint operators $A$ on a Hilbert space $\mathscr{H}$, and a quantum mechanical system is described by the von Neumann algebra $\mathscr{A}$ generated by its observables. A normal state $\rho \in \mathscr{S}(\mathscr{A})$ on $\mathscr{A}$ induces a probability measure on the $\operatorname{spectrum} \operatorname{Spec}(A)$ of an observable $A$, and it is the

\footnotetext{
Bas Janssens

janssens@mpim-bonn.mpg.de

1 Max Planck Institute for Mathematics, Vivatsgasse 7, 53111 Bonn, Germany
} 
objective of a quantum measurement to portray this probability measure as faithfully as possible.

For the more detailed results of Sects. 5 and 6, we will assume that $\mathscr{A}$ is the algebra $B(\mathscr{H})$ of all bounded operators on a separable Hilbert space $\mathscr{H}$, an assumption which is justified for quantum systems consisting of finitely many particles. In this case, all normal states on $\mathscr{A}$ are of the form $\rho(A):=\operatorname{tr}(r A)$, where $r \in \mathscr{T}_{1,+}(\mathscr{H})$ is a positive normalized trace class operator on $\mathscr{H}$, the density operator.

According to the uncertainty relation $\sigma_{X} \sigma_{Y} \geq \frac{1}{2}|\rho([X, Y])|$, (see $\left.[5,14,26]\right)$, there is an inherent variance in the quantum state. Furthermore, quantum theory puts severe restrictions on the performance of measurement. These restrictions, which come on top of the measurement restrictions implied by the above uncertainty relation, fall into three distinct classes.

(I) The impossibility of perfect joint measurement. It is not possible to perform a simultaneous measurement of two noncommuting observables in such a way that both measurements have perfect quality.

(II) The Heisenberg Principle, (see [5]). In this paper, this means that quantum information cannot be extracted from a system without disturbing that system. (There are various other formulations of the Heisenberg Principle in the literature, but throughout the paper, it will exclusively have the above meaning.)

(III) The collapse of the wave function. When information is extracted from a quantum system, a so-called decoherence is experimentally known to occur on this system.

We will see that this collapse of the wave function is a mathematical consequence of information extraction. In the process, II and III will be clearly exhibited as two sides of the same coin.

The subject of uncertainty relations in quantum measurement is already endowed with an extensive literature. For example, the Heisenberg Principle and the impossibility of joint measurement are quantitatively illustrated in $[2,4,8,22]$.

However, the inequalities in these papers depend on the state $\rho$, which somewhat limits their practical use. Indeed, the bound on the measurement quality can only be calculated if the state $\rho$ is known, in which case there is no need for a measurement in the first place.

Our state-independent figures of merit (Sects. 2, 3) will lead us quite naturally to state-independent bounds on the performance of measurement. In order to illustrate their practical use, we will give some applications. We investigate the beamsplitter, resonance fluorescence and nondestructive qubit measurement.

In Sect. 4, we will prove a sharp, state-independent bound on the performance of jointly unbiased measurement. This generalizes the impossibility of perfect joint measurement.

In Sect. 5, we will prove a sharp, state-independent bound on the performance of a measurement in terms of the maximal disturbance that it causes. This generalizes the Heisenberg Principle.

In contrast to the Heisenberg Principle and its abundance of inequalities, the phenomenon of decoherence has mainly been investigated in specific examples (see, e.g. $[6,11,32])$. Although there are some bounds on the remaining coherence in terms of 
the measurement quality (see [10,27]), a sharp, information-theoretic inequality does not yet appear to exist.

We will provide such an inequality in Sect. 6, where we will prove a sharp upper bound on the amount of coherence which can survive information transfer. Not only does this generalize the collapse of the wave function, it also shows that no information can be extracted if all coherence is left perfectly intact. It is therefore a unified description of both the Heisenberg Principle and the collapse of the wave function.

\section{Information transfer}

In quantum mechanics, a system is described by a von Neumann algebra $\mathscr{A}$ of bounded operators on a Hilbert space $\mathscr{H}$. The space $\mathscr{S}(\mathscr{A})$ of normal states of the system $\mathscr{A}$ is formed by the linear functionals $\rho: \mathscr{A} \rightarrow \mathbb{C}$ which are positive (i.e. $\rho\left(A^{\dagger} A\right) \geq 0$ for $A \in \mathscr{A}$ ), normalized (i.e. $\rho(\mathbf{1})=1$ ) and normal (i.e. weakly continuous on the unit ball $\left.\mathscr{A}_{1}\right)$. If $\mathscr{A}$ is the algebra $B(\mathscr{H})$ of all bounded operators on a separable Hilbert space $\mathscr{H}$, then every normal state on $\mathscr{A}$ is of the form $\rho(A):=\operatorname{tr}(r A)$, with $r \in \mathscr{T}_{1,+}(\mathscr{H})$ a positive normalized $(\operatorname{tr}(r)=1)$ trace class operator on $\mathscr{H}$ (cf. [13, Ch. 7]), the density operator. With the system in state $\rho \in \mathscr{S}(\mathscr{A})$, observation of an (Hermitean) observable $A \in \mathscr{A}$ is postulated to yield the average value $\rho(A)$.

Definition 1 Let $\mathscr{A}$ and $\mathscr{B}$ be von Neumann algebras. A map $T: \mathscr{B} \rightarrow \mathscr{A}$ is called Completely Positive (or CP for short) if it is linear, normalized (i.e. $T(\mathbf{1})=\mathbf{1}$ ), positive (i.e. $T\left(X^{\dagger} X\right) \geq 0$ for all $X \in \mathscr{B}$ ) and if, moreover, the extension $\operatorname{id}_{n} \otimes T: M_{n} \otimes \mathscr{B} \rightarrow$ $M_{n} \otimes \mathscr{A}$ is positive for all $n \in \mathbb{N}$, where $M_{n}$ is the algebra of complex $n \times n$-matrices. In this paper, we will require $\mathrm{CP}$-maps to be weakly continuous on the unit ball $\mathscr{B}_{1}$ unless specified otherwise.

Its dual $T^{*}: \mathscr{S}(\mathscr{A}) \rightarrow \mathscr{S}(\mathscr{B})$, defined by $T^{*}(\rho):=\rho \circ T$, has a direct physical interpretation as an operation between quantum systems. First of all, due to positivity and normalization of $T$, each state $\rho \in \mathscr{S}(\mathscr{A})$ is again mapped to a state $T^{*}(\rho) \in$ $\mathscr{S}(\mathscr{B})$. Secondly, linearity implies that $T^{*}$ satisfies

$$
p T^{*}\left(\rho_{1}\right)+(1-p) T^{*}\left(\rho_{2}\right)=T^{*}\left(p \rho_{1}+(1-p) \rho_{2}\right)
$$

for all $p \in[0,1], \rho_{1}, \rho_{2} \in \mathscr{S}(\mathscr{A})$. This expresses the stochastic equivalence principle: a system which is in state $\rho_{1}$ with probability $p$ and in state $\rho_{2}$ with probability $(1-p)$ cannot be distinguished from a system in state $p \rho_{1}+(1-p) \rho_{2}$. Finally, it is possible to extend the systems $\mathscr{A}$ and $\mathscr{B}$ under consideration with another system $M_{n}$, on which the operation acts trivially. Due to complete positivity, states in $\mathscr{S}\left(M_{n} \otimes \mathscr{A}\right)$ are once again mapped to states in $\mathscr{S}\left(M_{n} \otimes \mathscr{B}\right)$. Incidentally, any CP-map $T$ automatically satisfies $T\left(X^{\dagger}\right)=T(X)^{\dagger}$ and $\|T(X)\| \leq\|X\|$ for all $X \in \mathscr{B}$.

\subsection{General, unbiased and perfect information transfer}

Suppose that we are interested in the distribution of the observable $A \in \mathscr{A}$, with the system $\mathscr{A}$ in some unknown state $\rho$. We perform the operation $T^{*}: \mathscr{S}(\mathscr{A}) \rightarrow \mathscr{S}(\mathscr{B})$ 
and then observe the 'pointer' $B$ in $\mathscr{B}$ in order to obtain information on $A$. One may (see [4]) take the position that any CP-map $T: \mathscr{B} \rightarrow \mathscr{A}$ is an information transfer from any observable $A \in \mathscr{A}$ to any pointer $B \in \mathscr{B}$. The following is a figure of demerit for the quality of such an information transfer.

Definition 2 Let $T: \mathscr{B} \rightarrow \mathscr{A}$ be a CP-map. Its measurement infidelity $\delta$ in transferring information from $A$ to the pointer $B$ is defined as

$$
\delta:=\sup _{S}\left\|\mathbf{1}_{S}(A)-T\left(\mathbf{1}_{S}(B)\right)\right\|,
$$

where $S$ runs over the Borel subsets of $\mathbb{R}$.

It measures how accurately probability distributions on the measured observable $A$ are copied to the pointer $B$.

The initial state $\rho$ defines a probability distribution $\mathbb{P}_{i}$ on the spectrum of $A$ by $\mathbb{P}_{i}(S):=\rho\left(\mathbf{1}_{S}(A)\right)$, where $\mathbf{1}_{S}(A)$ denotes the spectral projection of $A$ associated with the set $S$. Similarly, the final state $T^{*}(\rho)$ defines a probability distribution $\mathbb{P}_{f}$ on the spectrum of $B . \delta$ is now the maximum distance between $\mathbb{P}_{i}$ and $\mathbb{P}_{f}$, where the maximum is taken over all initial states $\rho$. That is, $\delta=\sup _{\rho} D\left(\mathbb{P}_{i}, \mathbb{P}_{f}\right)$.

The trace distance (a.k.a. variational distance or Kolmogorov distance) is defined as

$$
D\left(\mathbb{P}_{f}, \mathbb{P}_{i}\right):=\sup _{S}\left\{\left|\mathbb{P}_{i}(S)-\mathbb{P}_{f}(S)\right|\right\}
$$

the difference between the probability that the event $S$ occurs in the distribution $\mathbb{P}_{i}$ and the probability that it occurs in the distribution $\mathbb{P}_{f}$, for the worst-case Borel set $S$. Writing out this definition, we see that indeed

$$
\begin{aligned}
\sup _{\rho} D\left(\mathbb{P}_{i}, \mathbb{P}_{f}\right) & =\sup _{\rho, S}\left|\rho\left(\mathbf{1}_{S}(A)\right)-\rho\left(T\left(\mathbf{1}_{S}(B)\right)\right)\right| \\
& =\sup _{S}\left\|\mathbf{1}_{S}(A)-T\left(\mathbf{1}_{S}(B)\right)\right\|,
\end{aligned}
$$

which equals $\delta$. The measurement infidelity $\delta$ is thus precisely the worst-case difference between input and output probabilities.

In this paper, we will devote considerable attention to the class of unbiased information transfers.

Definition 3 A CP-map $T: \mathscr{B} \rightarrow \mathscr{A}$ is called an unbiased information transfer from the Hermitean observable $A \in \mathscr{A}$ to a Hermitean $B \in \mathscr{B}$ if $T(B)=A$.

Recall that we are interested in the distribution of $A$, with the system $\mathscr{A}$ in some unknown state $\rho \in \mathscr{S}(\mathscr{A})$. We perform the operation $T^{*}: \mathscr{S}(\mathscr{A}) \rightarrow \mathscr{S}(\mathscr{B})$ and then observe the 'pointer' $B$ in $\mathscr{B}$. Since $T^{*}(\rho)(B)=\rho(T(B))$ by definition of the dual, and $\rho(T(B))=\rho(A)$ by definition of unbiased information transfer, the expectation value of $B$ in the final state $T^{*}(\rho)$ is the same as that of $A$ in the initial state $\rho$. We conclude that the expectation of $A$ was transferred to $B$. 
Definition 4 An information transfer $T: \mathscr{B} \rightarrow \mathscr{A}$ from $A \in \mathscr{A}$ to $B \in \mathscr{B}$ is called perfect if $\delta=0$. Equivalently, it is perfect if $T(B)=A$, and if the restriction of $T$ to $B^{\prime \prime}$, the von Neumann algebra generated by $B$, is a *homomorphism $B^{\prime \prime} \rightarrow A^{\prime \prime}$.

The entire probability distribution of $A$ is then transferred to $B$, rather than merely its average value. Indeed, for all moments $\rho\left(A^{n}\right)$, we have $T^{*}(\rho)\left(B^{n}\right)=\rho\left(T\left(B^{n}\right)\right)=$ $\rho\left(T(B)^{n}\right)=\rho\left(A^{n}\right)$. Everything there is to know about $A$ in the initial state $\rho$ can be obtained by observing the 'pointer' $B$ in the final state $T^{*}(\rho)$.

Between the different kinds of information transfer (IT), the following relations hold:

$\{$ General IT $\} \supset\{$ Unbiased IT $\} \supset\{$ Perfect IT $\}$.

\subsection{Example: von Neumann qubit measurement}

Let $\Omega:=\{+1,-1\}$. Denote by $\mathscr{C}(\Omega)$ the (commutative) algebra of $\mathbb{C}$-valued random variables on $\Omega$. A state on $\mathscr{C}(\Omega)$ is precisely the expectation value $\mathbb{E}$ w.r.t. a probability distribution $\mathbb{P}$ on $\Omega$, in which context $\rho(f)$ is denoted $\mathbb{E}(f)$. Define the probability distributions $\mathbb{P}_{ \pm}$to assign probability 1 to \pm 1 .

The von Neumann measurement $T: M_{2} \otimes \mathscr{C}(\Omega) \rightarrow M_{2}$ on a qubit (described by the algebra $M_{2}$ of complex $2 \times 2$ matrices) is defined as

$$
T(X \otimes f):=f(+1) P_{+} X P_{+}+f(-1) P_{-} X P_{-},
$$

with $P_{+}=|\uparrow\rangle\langle\uparrow|$ and $P_{-}=|\downarrow\rangle\langle\downarrow|$. Then $T^{*}: \mathscr{S}\left(M_{2}\right) \rightarrow \mathscr{S}\left(M_{2}\right) \otimes \mathscr{S}(\mathscr{C}(\Omega))$ is given by

$$
T^{*}(\rho)=\rho\left(P_{+}\right)|\uparrow\rangle\left\langle\uparrow\left|\otimes \mathbb{P}_{+}+\rho\left(P_{-}\right)\right| \downarrow\right\rangle\langle\downarrow| \otimes \mathbb{P}_{-} .
$$

In words: with probability $\rho\left(P_{+}\right)$, the output +1 occurs and the qubit is left in state $|\uparrow\rangle$. With probability $\rho\left(P_{-}\right)$, the output -1 occurs, leaving the qubit in state $|\downarrow\rangle$. The von Neumann measurement $T$ is a perfect (and thus unbiased) information transfer from $\sigma_{z} \in M_{2}$ to the pointer $1 \otimes\left(\delta_{+1}-\delta_{-1}\right) \in M_{2} \otimes \mathscr{C}(\Omega)$.

\subsection{CP-maps and POVMs}

Quantum measurements are often (e.g. [4,7]) modelled by positive operator-valued measures or POVMs. From the above CP-map $T$, we may distil the POVM $\mu: \Omega \rightarrow M_{2}$ by $\mu(\omega):=T\left(1 \otimes \delta_{\omega}\right)$, i.e. $\mu(+1)=P_{+}$and $\mu(-1)=P_{-}$.

This procedure is fully general: given a CP-map $T: \mathscr{B} \rightarrow \mathscr{A}$ and a 'pointer' $B \in \mathscr{B}$, we obtain an $\mathscr{A}$-valued POVM $\mu_{B, T}$ on $\operatorname{Spec}(B)$ by $\mu_{T, B}(S):=T\left(\mathbf{1}_{S}\right)$. (This is why we require CP-maps to be weakly continuous on the unit ball $\mathscr{B}_{1}$.) Conversely, any $\mathscr{A}$-valued POVM $\mu$ on $\Omega$ gives rise to the CP-map $T_{\mu}: L^{\infty}(\Omega) \rightarrow \mathscr{A}$ by integration, $T_{\mu}(f)=\int_{\Omega} f(\omega) \mu(d \omega)$. 
A CP-map can thus be seen as an extension of a POVM that keeps track of the system output as well as the measurement output. Since we will be interested in disturbance of the system, it is imperative that we consider the full CP-map rather than merely its POVM.

\section{Maximal added variance}

For unbiased information transfer, there exists a figure of demerit more attractive than $\delta$. Consider the variance $\operatorname{Var}\left(B, T^{*}(\rho)\right)$ of the output, where the variance of $X$ in the state $\rho$ is defined as

$$
\operatorname{Var}(X, \rho):=\rho\left(X^{\dagger} X\right)-\rho(X)^{*} \rho(X)
$$

The output variance can be split into two parts. One part $\operatorname{Var}(A, \rho)$ is the variance of the input, which is intrinsic to the quantum state $\rho$. The other $\operatorname{part} \operatorname{Var}\left(B, T^{*}(\rho)\right)-$ $\operatorname{Var}(A, \rho) \geq 0$ is added by the measurement procedure. This second part determines how well the measurement performs.

The maximal added variance (where the maximum is taken over the input states $\rho$ ) will be our figure of demerit. For example, perfect information transfer from $A$ to $B$ satisfies $\operatorname{Var}\left(B, T^{*}(\rho)\right)=\operatorname{Var}(A, \rho)$, so that the maximal added variance is 0 . There is uncertainty in the measurement outcome, but all uncertainty 'comes from' the quantum state, and none is added by the measurement procedure.

Definition 5 The maximal added variance of an unbiased information transfer $T$ is defined as

$$
\Sigma^{2}:=\sup _{\rho \in \mathscr{S}(\mathscr{A})} \operatorname{Var}\left(B, T^{*}(\rho)\right)-\operatorname{Var}(A, \rho) .
$$

It is straightforward to verify that $\Sigma^{2}=\left\|T\left(B^{\dagger} B\right)-T(B)^{\dagger} T(B)\right\|$. This inspires the following definition.

Definition 6 Let $T: \mathscr{B} \rightarrow \mathscr{A}$ be a CP-map. We define the operator-valued sesquilinear form $(\cdot, \cdot): \mathscr{B} \times \mathscr{B} \rightarrow \mathscr{A}$ by

$$
(X, Y):=T\left(X^{\dagger} Y\right)-T(X)^{\dagger} T(Y) \text {. }
$$

It satisfies $(X, Y)^{\dagger}=(Y, X)$ and is positive semi-definite: $(B, B) \geq 0$ for all $B \in \mathscr{B}$ (cf. also [12]). This 'length' has the physical interpretation $\|(B, B)\|=\Sigma^{2}$, and there is even a Cauchy-Schwarz inequality:

Lemma 1 (Cauchy-Schwarz) Let $T: \mathscr{B} \rightarrow \mathscr{A}$ be a CP-map, and $(X, Y):=$ $T\left(X^{\dagger} Y\right)-T(X)^{\dagger} T(Y)$. Then for all $X, Y \in \mathscr{B}$ :

$$
(X, Y)(Y, X) \leq\|(Y, Y)\|(X, X) .
$$


Proof By Stinespring's theorem (see [28]), we may assume without loss of generality that $T$ is of the form $T(X)=V^{\dagger} X V$ for some contraction $V$. Writing this out, we obtain $(X, Y)=V^{\dagger} X^{\dagger}\left(\mathbf{1}-V V^{\dagger}\right) Y V$. Defining $g(X):=\sqrt{\mathbf{1}-V V^{\dagger}} X V$, we have $(X, Y)=g(X)^{\dagger} g(Y)$. Hence $(X, Y)(Y, X)=g(X)^{\dagger} g(Y) g(Y)^{\dagger} g(X)$, which is less or equal than $\|g(Y)\|^{2} g(X)^{\dagger} g(X)=\|(Y, Y)\|(X, X)$.

If an information transfer is perfect, then of course $\Sigma^{2}=\|(B, B)\|=0$. (No variance is added.) We will now show that the converse also holds: if $\Sigma^{2}=0$, then $T$ is a *-homomorphism on $B^{\prime \prime}$. (Compare this with the fact that probability distributions of zero variance are concentrated in a single point.)

Theorem 1 Let $T: \mathscr{B} \rightarrow \mathscr{A}$ be a CP-map, and let $B \in \mathscr{B}$ be Hermitean. Then among

1. $(B, B)=0$.

2. The restriction of $T$ to $B^{\prime \prime}$, the von Neumann algebra generated by $B$, is a *-homomorphism $B^{\prime \prime} \rightarrow T(B)^{\prime \prime}$.

3. $(f(B), f(B))=0$ for all measurable functions $f$ on the spectrum of $B$.

4. T maps the relative commutant $B^{\prime}=\{X \in \mathscr{A} ;[X, B]=0\}$ into $T(B)^{\prime}$.

the following relations hold: (1) $\Leftrightarrow(2) \Leftrightarrow(3) \Rightarrow$ (4).

Proof For (1) $\Rightarrow$ (2), use Cauchy-Schwarz (Lemma 1) to find $T\left(B^{n}\right)-T(B) T\left(B^{n-1}\right)$ $\leq\|(B, B)\|\left(B^{n-1}, B^{n-1}\right)=0$. By induction, we have $T\left(B^{n}\right)=T(B)^{n}$, and by linearity $T(f(B))=f(T(B))$ for all polynomials $f$. Thus, $T$ is a *homomorphism from the algebra of polynomials on the spectrum of $B$ to that on $T(B)$. Since $T$ is positive, it is norm continuous, hence extends to a $C^{*}$-algebra homomorphism between the algebras $C(\operatorname{Spec}(B))$ and $C(\operatorname{Spec}(A))$ of continuous functions on the spectra. Moreover, since we require $\mathrm{CP}$-maps to be continuous in the weak operator topology on the unit ball and since every measurable function on the spectrum can be approximated weakly by a uniformly bounded sequence of continuous functions, the statement even extends to the algebras of measurable functions on the spectra of $B$ and $T(B)$, isomorphic to $B^{\prime \prime}$ and $T(B)^{\prime \prime}$, respectively. For (2) $\Rightarrow$ (3), note that $T\left(f(B)^{2}\right)=T(f(B))^{2}$. For $(3) \Rightarrow(1)$, take $f(x)=x$. Finally, we prove $(1) \Rightarrow(4)$. Suppose that $[A, B]=0$. Then $[T(B), T(A)]=T([A, B])-[T(A), T(B)]=$ $\left(A^{\dagger}, B\right)-\left(B^{\dagger}, A\right)(B$ is Hermitean). By Cauchy-Schwarz, the last term equals zero if $(B, B)$ does.

We see that the maximal added variance $\Sigma^{2}$ equals 0 if and only if $T$ is a perfect information transfer. We shall take $\Sigma$ to parametrize the imperfection of unbiased information transfer.

\section{Joint measurement}

In a jointly unbiased measurement, information on two observables $A$ and $\tilde{A}$ is transferred to two commuting pointers $B$ and $\tilde{B}$. If $A$ and $\tilde{A}$ do not commute, then it is not possible for both information transfers to be perfect. (See [29,30].) Indeed, the degree of imperfection is determined by the amount of noncommutativity. 
Let $T: \mathscr{B} \rightarrow \mathscr{A}$ be a CP-map, and let $B, \tilde{B} \in \mathscr{B}$ be Hermitean observables in $\mathscr{B}$. Set $A:=T(B), \tilde{A}:=T(\tilde{B}), \Sigma_{B}^{2}:=\|(B, B)\|$, and $\Sigma_{\tilde{B}}^{2}:=\|(\tilde{B}, \tilde{B})\|$.

Theorem 2 If $B$ and $\tilde{B}$ commute, then

$$
\Sigma_{B} \Sigma_{\tilde{B}} \geq \frac{1}{2}\|[A, \tilde{A}]\|
$$

Proof Since $[B, \tilde{B}]=0$, we have $[\tilde{A}, A]=T([B, \tilde{B}])-[T(B), T(\tilde{B})]=(B, \tilde{B})-$ $(\tilde{B}, B)$. By Cauchy-Schwarz, the latter is at most $2 \Sigma_{B} \Sigma_{\tilde{B}}$ in norm.

Remark 1 The $\mathscr{A}$-valued POVMs $\mu_{i}$ on $\Omega_{i} \subseteq \mathbb{R}$ are called jointly measurable (e.g. $\left[15, \S 2\right.$ and 7]) if there exists a POVM $\mu$ on $\Omega$ and measurable functions $B_{i}: \Omega \rightarrow \Omega_{i}$ such that $\mu_{i}=\mu \circ B_{i}^{-1}$. If the sets $\Omega_{i}$ are bounded, we can apply Theorem 2 to the CP-map $T_{\mu}: L^{\infty}(\Omega) \rightarrow \mathscr{A}$ obtained by integrating against $\mu$. Since $\Sigma_{B_{i}, T_{\mu}}^{2}=$ $\Sigma_{x, T_{\mu_{i}}}^{2}$, we find $\Sigma_{x, T_{\mu_{i}}} \cdot \Sigma_{x, T_{\mu_{j}}} \geq \frac{1}{2}\left\|\left[A_{i}, A_{j}\right]\right\|$. Here, $\Sigma_{x, T_{\mu_{i}}}^{2}=\| \int_{\Omega_{i}} x^{2} \mu_{i}(d x)-$ $\left(\int_{\Omega_{i}} x \mu_{i}(d x)\right)^{2} \|$ is the maximal added variance of $T_{\mu_{i}}$ as an unbiased measurement of $A_{i}:=\int_{\Omega_{i}} x \mu_{i}(d x)$.

We now show that the bound (1) is sharp in the sense that for all $S, \tilde{S}>0$, there exist $T, B, \tilde{B}$ such that (1) attains equality with $\Sigma_{B}=S, \Sigma_{\tilde{B}}=\tilde{S}$.

\subsection{Application: The beamsplitter as a joint measurement}

A beamsplitter is a device which takes two beams of light as input. A certain fraction of each incident beam is refracted and the rest is reflected, in such a way that the refracted part of the first beam coincides with the reflected part of the second and vice versa (cf. Fig. 1). We will show that the beamsplitter serves as an optimal joint unbiased measurement.

In cavity QED, a single mode in the field is described by a Hilbert space $\mathscr{H}$ of a harmonic oscillator, with creation and annihilation operators $a^{\dagger}$ and $a$ satisfying $\left[a, a^{\dagger}\right]=1$, as well as $x=\frac{a+a^{\dagger}}{\sqrt{2}}$ and $p=\frac{a-a^{\dagger}}{\sqrt{2} i}$. The coherent states $|\alpha\rangle=e^{-|\alpha|^{2} / 2} \sum_{n=0}^{\infty} \frac{\alpha^{n}}{\sqrt{n !}}|n\rangle$ are dense in $\mathscr{H}$, and satisfy $a|\alpha\rangle=\alpha|\alpha\rangle$.

Quantummechanically, a beamsplitter is described by the unitary operator $U$ on $\mathscr{H} \otimes \mathscr{H}$, given by $U=\exp \left(\theta\left(a^{\dagger} \otimes a-a \otimes a^{\dagger}\right)\right)$. In terms of the coherent vectors, we have $U|\alpha\rangle \otimes|\beta\rangle=|\alpha \cos (\theta)+\beta \sin (\theta)\rangle \otimes|-\alpha \sin (\theta)+\beta \cos (\theta)\rangle$. Note that

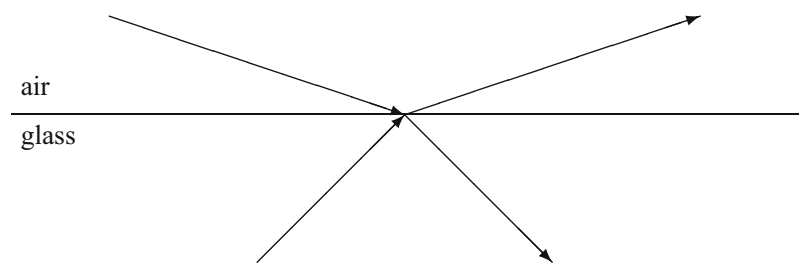

Fig. 1 Beamsplitter 
$U^{\dagger} a \otimes \mathbf{1} U=\cos (\theta) a \otimes \mathbf{1}+\sin (\theta) \mathbf{1} \otimes a$ and that $U^{\dagger} \mathbf{1} \otimes a U=-\sin (\theta) a \otimes \mathbf{1}+$ $\cos (\theta) \mathbf{1} \otimes a$. (This can be seen by sandwiching both sides between coherent vectors.) Since the map $Y \mapsto U^{\dagger} Y U$ respects,$+ \cdot$ and ${ }^{\dagger}$, we readily calculate

$$
\begin{aligned}
U^{\dagger} x \otimes \mathbf{1} U & =\cos (\theta) x \otimes \mathbf{1}+\sin (\theta) \mathbf{1} \otimes x, \\
U^{\dagger} x^{2} \otimes \mathbf{1} U & =\cos ^{2}(\theta) x^{2} \otimes \mathbf{1}+2 \sin (\theta) \cos (\theta) x \otimes x+\sin ^{2}(\theta) \mathbf{1} \otimes x^{2}, \\
U^{\dagger} \mathbf{1} \otimes p U & =-\sin (\theta) p \otimes \mathbf{1}+\cos (\theta) \mathbf{1} \otimes p, \\
U^{\dagger} \mathbf{1} \otimes p^{2} U & =\sin ^{2}(\theta) p^{2} \otimes \mathbf{1}-2 \cos (\theta) \sin (\theta) p \otimes p+\cos ^{2}(\theta) \mathbf{1} \otimes p^{2} .
\end{aligned}
$$

Since the von Neumann algebra describing a single mode in the field is $B(\mathscr{H})$, we identify the normal state space $\mathscr{S}(B(\mathscr{H}))$ with the space $\mathscr{T}_{1,+}(\mathscr{H})$ of positive normalized trace class operators, and we are interested in the map $r \mapsto U r \otimes|0\rangle\langle 0| U^{\dagger}$ from $\mathscr{T}_{1,+}(\mathscr{H})$ to $\mathscr{T}_{1,+}(\mathscr{H}) \otimes \mathscr{T}_{1,+}(\mathscr{H})$. In other words, we feed the beamsplitter only one beam of light in a state $\rho_{r}$ described by the density operator $r$, the other input being the vacuum. The dual of this is the CP-map $T: \mathscr{B}(\mathscr{H}) \otimes \mathscr{B}(\mathscr{H}) \rightarrow \mathscr{B}(\mathscr{H})$ defined by $T(Y):=$ id $\otimes \phi_{0}\left(U^{\dagger} Y U\right)$, with $\phi_{0}$ the vacuum state $\phi_{0}(X)=\langle 0|X| 0\rangle$.

Take $B=\cos ^{-1}(\theta) x \otimes \mathbf{1}$ for instance. Then $T(B)=x\langle 0|\mathbf{1}| 0\rangle+\tan (\theta) \mathbf{1}\langle 0|x| 0\rangle=x$. Similarly, with $\tilde{B}=-\sin ^{-1}(\theta) \mathbf{1} \otimes p$, we have $T(\tilde{B})=p$. Apparently, splitting a beam of light in two parts, measuring $x \otimes \mathbf{1}$ in the first beam and $\mathbf{1} \otimes p$ in the second, and then compensating for the loss of intensity provides a simultaneous unbiased measurement of $x$ and $p$ in the original beam. Since $[x, p]=i$, we must ${ }^{1}$ have $\Sigma_{B} \Sigma_{\tilde{B}} \geq \frac{1}{2}$.

We now calculate $\Sigma_{B}$ and $\Sigma_{\tilde{B}}$ explicitly. From $\left\langle 0\left|x^{2}\right| 0\right\rangle=\frac{1}{2}$, we see that $T\left(B^{2}\right)=$ $x^{2}+\frac{1}{2} \tan ^{2}(\theta)$ 1. Thus, $\Sigma_{B}^{2}=\|(B, B)\|=\frac{1}{2} \tan ^{2}(\theta)$. Similarly, $\Sigma_{\tilde{B}}^{2}=\frac{1}{2} \tan ^{-2}(\theta)$. We see that $\Sigma_{B} \Sigma_{\tilde{B}}=\frac{1}{2}$, so that the beamsplitter is indeed an optimal jointly unbiased measurement.

By scaling $B$, optimal joint measurements can be found for arbitrary values of $\Sigma_{B}$ and $\Sigma_{\tilde{B}}$, which shows the bound in Theorem 2 to be sharp. It may therefore be used to evaluate joint measurement procedures. For example, it was shown in [9] that homodyne detection of the spontaneous decay of a two-level atom constitutes a joint measurement with $\Sigma \Sigma^{\prime}=1.056$, slightly above the bound $\Sigma \Sigma^{\prime} \geq 1$ provided by Theorem 2.

The beamsplitter is an optimal joint measurement in the sense that it minimizes $\Sigma \Sigma^{\prime}$. It also performs well with other figures of merit. For example, if the quality of joint measurement is judged by the state-dependent $\operatorname{cost} R(T):=\operatorname{Var}\left(B, T^{*}(\rho)\right)+$ $\operatorname{Var}\left(\tilde{B}, T^{*}(\rho)\right)$, then at least for Gaussian $\rho$, the optimal measurement is again the above beamsplitter with $\theta=\pi / 4$. (See [7].)

\section{The Heisenberg Principle}

The Heisenberg Principle may be stated as follows:

\footnotetext{
${ }^{1}$ We neglect the technical complication of $x$ and $p$ being unbounded operators.
} 
If all states are left intact, no quantum information can be extracted from a system.

This alludes to an information transfer from an initial system $\mathscr{A}$ to a final system consisting of two parts: the system $\mathscr{A}$ and an ancilla $\mathscr{B}$, containing the pointer $B$. We thus have an information transfer $T: \mathscr{A} \otimes \mathscr{B} \rightarrow \mathscr{A}$ from $A$ to $\mathbf{1} \otimes B$.

An initial state $\rho \in \mathscr{S}(\mathscr{A})$ gives rise to a final state $T^{*}(\rho) \in \mathscr{S}(\mathscr{A} \otimes \mathscr{B})$. Restricting this final state to the system $\mathscr{A} \simeq \mathscr{A} \otimes \mathbf{1} \subseteq \mathscr{A} \otimes \mathscr{B}$ (this is called taking the partial trace over $\mathscr{B}$ ) yields a 'residual' state $R^{*}(\rho) \in \mathscr{S}(\mathscr{A})$, whereas taking the partial trace over $\mathscr{A}$ yields the final state $Q^{*}(\rho) \in \mathscr{S}(\mathscr{B})$ of the ancilla. We define the CP-maps $R: \mathscr{A} \rightarrow \mathscr{A}$ by $R(A):=T(A \otimes \mathbf{1})$ and $Q: \mathscr{B} \rightarrow \mathscr{A}$ by $Q(B):=T(1 \otimes B)$. The map $R$ describes what happens to $\mathscr{A}$ if we forget about the ancilla $\mathscr{B}$, and $Q$ describes the ancilla, neglecting the original system $\mathscr{A}$.

We wish to find a quantitative version of the Heisenberg Principle, i.e. we want to relate the imperfection of the extracted quantum information to the amount of state disturbance.

Definition 7 The maximal disturbance $\Delta$ of a map $R: \mathscr{A} \rightarrow \mathscr{A}$ is given by

$$
\Delta:=\sup \left\{\|R(P)-P\| ; P \in \mathscr{A}, P^{2}=P^{\dagger}=P\right\} .
$$

The trace distance (or Kolmogorov distance) $D(\tau, \rho)$ is the maximal difference between the probability $\tau(P)$ that an event $P$ occurs in the state $\tau$, and the probability $\rho(P)$ that it occurs in the state $\rho$, for the worst-case event (projection operator) $P$. For short, $D(\tau, \rho):=\sup _{P}\{|\tau(P)-\rho(P)|\}$. If $\tau$ and $\rho$ correspond to density operators $t$ and $r$, then $D(\tau, \rho)=\frac{1}{2} \operatorname{tr}(|t-r|)$ (see, e.g. [21]).

The maximal disturbance $\Delta$ is now the worst-case distance between the input $\rho$ and the output $R^{*}(\rho)$, i.e. $\Delta=\sup \left\{D\left(\rho, R^{*}(\rho)\right) ; \rho \in \mathscr{S}(\mathscr{A})\right\}$. Indeed,

$$
\sup _{\rho}\left\{D\left(\rho, R^{*}(\rho)\right)\right\}=\sup _{\rho, P}\{\rho(P)-\rho(R(P))\}
$$

which equals $\sup _{P}\{\|R(P)-P\|\}=\Delta$.

\subsection{Heisenberg Principle for unbiased information transfer}

We first turn our attention to unbiased information transfer. The imperfection of the information is then captured in the maximal added variance $\Sigma^{2}$.

The Heisenberg Principle only holds for quantum information. Classical observables are contained in the centre $\mathscr{Z}=\{A \in \mathscr{A} ;[A, X]=0 \forall X \in \mathscr{A}\}$, whereas quantum observables are not. The degree in which an observable $A$ is 'quantum' is given by its distance to the centre $d(A, \mathscr{Z})=\inf _{Z \in \mathscr{Z}}\|A-Z\|$.

In the rest of the paper, we will take the algebra of observables to be $B(\mathscr{H})$ for some separable Hilbert space $\mathscr{H}$.

The centre is then simply $\mathbb{C} 1$. Since normal states $\rho \in \mathscr{S}(B(\mathscr{H}))$ are given by density operators $r \in \mathscr{T}_{1,+}$ as $\rho(A)=\operatorname{tr}(r A)$, the dual $T^{*}: \mathscr{S}(B(\mathscr{H})) \rightarrow \mathscr{S}\left(B\left(\mathscr{H}^{\prime}\right)\right)$ of 
a CP-map $T: B\left(\mathscr{H}^{\prime}\right) \rightarrow B(\mathscr{H})$ yields a map $\mathscr{T}_{1,+}(\mathscr{H}) \rightarrow \mathscr{T}_{1,+}\left(\mathscr{H}^{\prime}\right)$, which we denote by $T_{*}$. We then have

$$
T^{*}(\rho)(A)=\operatorname{tr}(r T(A))=\operatorname{tr}\left(T_{*}(r) A\right)
$$

Theorem 3 Let $T: B(\mathscr{H}) \otimes \mathscr{B} \rightarrow B(\mathscr{H})$ be a $C P$-map, let $B \in \mathscr{B}$ be Hermitean. Define $A:=T(\mathbf{1} \otimes B)$, and $\Sigma^{2}:=\|(\mathbf{1} \otimes B, \mathbf{1} \otimes B)\|$. Further, define $\Delta:=\sup _{P}\{\|R(P)-P\|\}$, with $R$ the restriction of $T$ to $B(\mathscr{H}) \otimes 1$. Then

$$
\Sigma \geq d(A, \mathscr{Z}) \frac{\frac{1}{2}-\Delta}{\sqrt{\Delta(1-\Delta)}} .
$$

This bound is sharp in the sense that for all $\Delta \in\left[0, \frac{1}{2}\right]$, there exist $T$ and A for which (2) attains equality.

Proof For the sharpness, see Sect. 6.5. As for the bound, we may assume $\Delta<\frac{1}{2}$, since inequality (2) is trivially satisfied otherwise. Denote the spectrum of $A$ by $\operatorname{Spec}(A)$. Let $x:=\sup (\operatorname{Spec}(A))$ and $y:=\inf (\operatorname{Spec}(A))$, so that $d(A, \mathscr{Z})=\frac{x-y}{2}$. Without loss of generality, assume that there exist normalized eigenvectors $\psi_{x}$ and $\psi_{y}$ satisfying $A \psi_{x}=x \psi_{x}$ and $A \psi_{y}=y \psi_{y}$. (If this is not the case, choose $x^{\prime}$ and $y^{\prime}$ in $\operatorname{Spec}(A)$ arbitrarily close to $x$ and $y$ and complete the proof using approximate eigenvectors.) Define $\psi_{-}:=\frac{1}{\sqrt{2}}\left(\psi_{x}+\psi_{y}\right), \tilde{B}:=\left|\psi_{-}\right\rangle\left\langle\psi_{-}\right|$and $\tilde{A}:=T(\tilde{B} \otimes \mathbf{1})$.

We thus have $\|[A, \tilde{B}]\|=d(A, \mathscr{Z})$. Since $\|\tilde{A}-\tilde{B}\| \leq \Delta$, we have $\|[A, \tilde{A}-\tilde{B}]\| \leq$ $2 \Delta d(A, \mathscr{Z})$. Then by the triangle inequality,

$$
\|[T(\mathbf{1} \otimes B), T(\tilde{B} \otimes \mathbf{1})]\|=\|[A, \tilde{B}]+[A, \tilde{A}-\tilde{B}]\| \geq d(A, \mathscr{Z})(1-2 \Delta),
$$

which brings us in a position to apply Theorem 2 to the commuting pointers $\tilde{B} \otimes \mathbf{1}$ and $\mathbf{1} \otimes B$. This yields

$$
2 \Sigma \sqrt{\|(\tilde{B} \otimes \mathbf{1}, \tilde{B} \otimes \mathbf{1})\|} \geq d(A, \mathscr{Z})(1-2 \Delta) .
$$

In order to estimate $\|(\tilde{B} \otimes \mathbf{1}, \tilde{B} \otimes \mathbf{1})\|$, we first prove that $\operatorname{Spec}(\tilde{A}) \subseteq[0, \Delta] \cup$ $[(1-\Delta), 1]$. Let $a \in \operatorname{Spec}(\tilde{A})$. Since $T$ is a contraction and $0 \leq \tilde{B} \leq 1$, we have $0 \leq$ $a \leq 1$. Without loss of generality, assume that there exists a normalized eigenvector $\psi_{a}$ such that $\tilde{A} \psi_{a}=a \psi_{a}$. (Again, if this is not the case, one may use approximate eigenvectors.) Decompose $\psi_{a}$ over the eigenspaces of $\tilde{B}$, i.e., write $\psi_{a}=\chi_{1}+\chi_{0}$, with $\chi_{1} \perp \chi_{0}, \tilde{B} \chi_{1}=\chi_{1}$ and $\tilde{B} \chi_{0}=0$. Then, we have

$$
(\tilde{A}-\tilde{B}) \psi_{a}=(a-1) \chi_{1}+a \chi_{0} .
$$

Since $\left\|\chi_{1}\right\|^{2}+\left\|\chi_{0}\right\|^{2}=1$, the inequality

$$
\Delta^{2} \geq\left\|(\tilde{A}-\tilde{B}) \psi_{a}\right\|^{2}=(a-1)^{2}\left\|\chi_{1}\right\|^{2}+a^{2}\left\|\chi_{0}\right\|^{2}
$$


Fig. 2 Combinations $(\Delta, \Sigma)$ below the curve are forbidden, those above are allowed. (With $d(A, \mathscr{Z})=1$.)

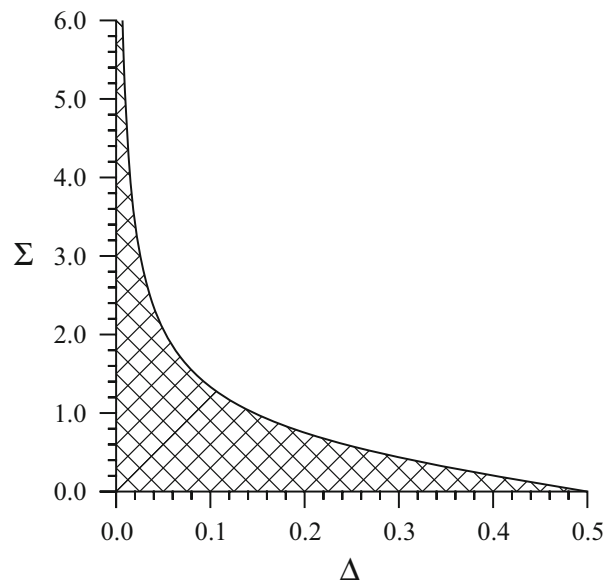

implies that either $|1-a| \leq \Delta$ or $a \leq \Delta$. Thus $\operatorname{Spec}(\tilde{A}) \subseteq[0, \Delta] \cup[(1-\Delta), 1]$, as desired. From this, it follows that $\operatorname{Spec}\left(\tilde{A}-\tilde{A}^{2}\right) \subseteq[0, \Delta(1-\Delta)]$. Since $\tilde{B}^{2}=\tilde{B}$, we may estimate

$$
\|(\tilde{B} \otimes \mathbf{1}, \tilde{B} \otimes \mathbf{1})\|=\left\|T(\tilde{B} \otimes \mathbf{1})-T(\tilde{B} \otimes \mathbf{1})^{2}\right\|=\left\|\tilde{A}-\tilde{A}^{2}\right\| \leq \Delta(1-\Delta) .
$$

Combining this with inequality (3) yields $2 \Sigma \sqrt{\Delta(1-\Delta)} \geq d(A, \mathscr{Z})(1-2 \Delta)$, which was to be demonstrated.

In the case of no disturbance, $\Delta=0$, we see that $\Sigma \rightarrow \infty$. No information transfer from $\mathscr{A}$ is allowed if all states on $\mathscr{A}$ are left intact. This is Werner's (see [31]) formulation of the Heisenberg Principle. In the opposite case of perfect information transfer, $\Sigma=0$, inequality 2 shows that $\Delta$ must equal at least one half. We shall see in Sect. 6 that this corresponds with a so-called collapse of the wave function.

These two extreme situations are connected by Theorem 3 in a continuous fashion, as indicated in Fig. 2. The upper left corner of the curve illustrates the Heisenberg Principle. In Sect. 6, we will see that the lower right corner represents the collapse of the wave function.

\subsection{Heisenberg Principle for general information transfer}

We now prove a version of the Heisenberg Principle for general information transfer. Let $T: B(\mathscr{H}) \otimes \mathscr{B} \rightarrow B(\mathscr{H})$ be a CP-map, and let $A \in B(\mathscr{H})$ and $B \in \mathscr{B}$ be Hermitean, with $A \notin \mathscr{Z}=\mathbb{C} 1$. Define $\Delta:=\sup _{P}\{\|R(P)-P\|\}$, with $R$ the restriction of $T$ to $B(\mathscr{H}) \otimes \mathbf{1}$. Define $\delta:=\sup _{S}\left\{\left\|T\left(\mathbf{1} \otimes \mathbf{1}_{S}(B)\right)-\mathbf{1}_{S}(A)\right\|\right\}$. In this setting, we find:

Corollary 1 For $\delta$ and $\Delta$ in $\left[0, \frac{1}{2}\right]$, we have

$$
\left(\frac{1}{2}-\delta\right)^{2}+\left(\frac{1}{2}-\Delta\right)^{2} \leq \frac{1}{4}
$$

This bound is sharp; for all $\Delta \in\left[0, \frac{1}{2}\right]$, there exists a $T$ for which (4) attains equality. 
Fig. 3 Combinations $(\Delta, \delta)$ below the curve are forbidden, those above are allowed

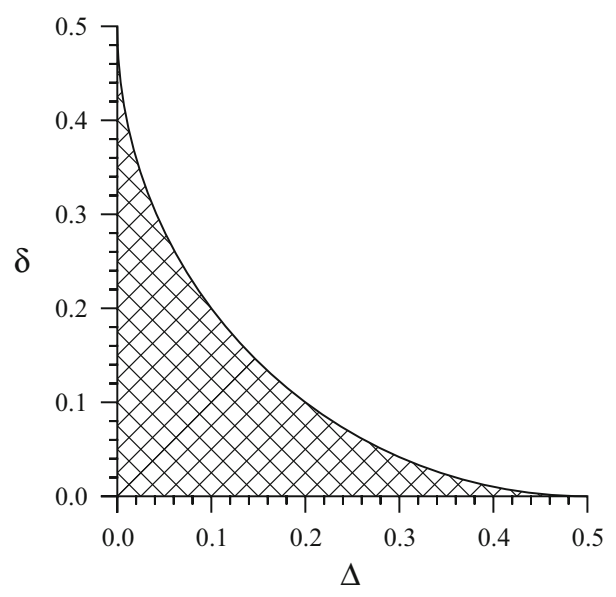

Proof Choose a nontrivial measurable subset $S$ of $\operatorname{Spec}(A)$, and put $P:=\mathbf{1} \otimes \mathbf{1}_{S}(B)$. Since $\left\|T(P)-\mathbf{1}_{S}(A)\right\| \leq \delta$ and $\operatorname{Spec}\left(\mathbf{1}_{S}(A)\right)=\{0,1\}$, we find that $\operatorname{Spec}(T(P)) \subseteq$ $[0, \delta] \cup[1-\delta, 1]$ (cf. the proof of Theorem 3). Thus $\Sigma^{2}=\left\|T(P)-T(P)^{2}\right\| \leq \delta(1-\delta)$. Similarly, $d(T(P), \mathscr{Z}) \geq \frac{1}{2}-\delta$ since $\operatorname{Spec}(T(P))$ contains points in both $[0, \delta]$ and $[1-\delta, 1]$. Applying Theorem 3 to the pointer $P$, we obtain

$$
\sqrt{\delta(1-\delta)} \geq\left(\frac{1}{2}-\delta\right)\left(\frac{1}{2}-\Delta\right) / \sqrt{\Delta(1-\Delta)}
$$

or equivalently $\left(\frac{1}{2}-\delta\right)^{2}+\left(\frac{1}{2}-\Delta\right)^{2} \leq \frac{1}{4}$. For sharpness, see Sect. 6.5.

A measurement that does not disturb any state $(\Delta=0)$ cannot yield information $\left(\delta \geq \frac{1}{2}\right)$. This is the Heisenberg Principle. On the other hand, perfect information ( $\delta=0)$ implies full disturbance $\left(\Delta \geq \frac{1}{2}\right.$ ), corresponding to the collapse of the wave function. Both extremes are connected in a continuous fashion, as depicted in Fig. 3.

\subsection{Application: Resonance fluorescence}

Corollary 1 may be used to determine the minimum amount of disturbance if the quality of the measurement is known. Alternatively, if the system is only mildly disturbed, one may find a bound on the attainable measurement quality. Let us concentrate on the latter option.

We investigate the radiation emission of a laser-driven two-level atom. The emitted EM radiation yields information on the atom. A two-level atom (i.e. a qubit) only has three independent observables: $\sigma_{x}, \sigma_{y}$ and $\sigma_{z}$. There are various ways to probe the EM field: photon counting, homodyne detection, heterodyne detection, etc. For a strong $(\Omega \gg 1)$ resonant $\left(\omega_{\text {laser }}=\omega_{\text {atom }}\right)$ laser, we will use Corollary 1 to prove 
Fig. 4 Lower bound on $\delta$ in terms of $t$ (in units of $\lambda^{-2}$ )

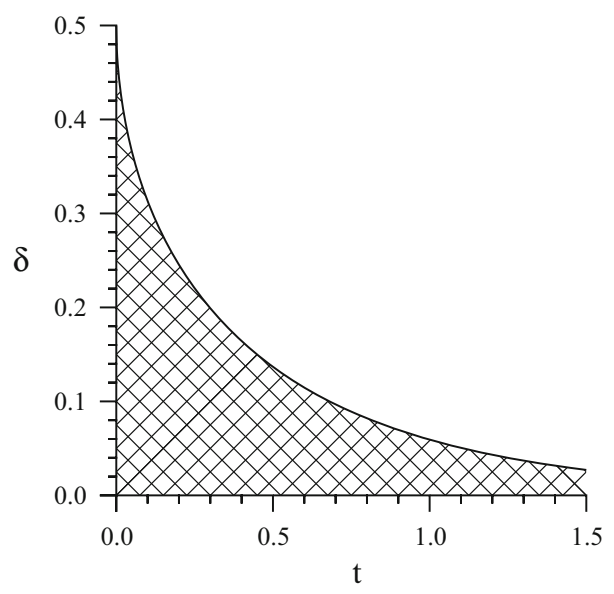

that any EM measurement of $\sigma_{x}, \sigma_{y}$ or $\sigma_{z}$ will have a measurement infidelity of at least

$$
\delta \geq \frac{1}{2}-\frac{1}{2} \sqrt{1-e^{-\frac{3}{2} \lambda^{2} t}}
$$

with $\lambda$ the coupling constant. For a measurement with two outcomes, $\delta$ is the maximal probability of getting the wrong outcome. (Cf. Fig. 4.)

\subsubsection{Unitary evolution on the closed system}

The atom is modelled by the Hilbert space $\mathbb{C}^{2}$ (only two energy levels are deemed relevant). In the field, we discern a forward and a side channel, each described by a bosonic Fock space $\mathscr{F}$. The laser is put on the forward channel, which is thus initially in the coherent state with frequency $\omega$ and strength $\Omega$, described by the density matrix $\phi_{\Omega}$. (The field strength is parametrized by the frequency of the induced Rabi oscillations). The side channel starts in the vacuum state, described by the density matrix $\phi_{0}$. If the two-level atom starts in the state described by the density matrix $r$, then the state at time $t$ is given by the density matrix

$$
T_{t *}(r)=U(t)\left(r \otimes \phi_{\Omega} \otimes \phi_{0}\right) U^{\dagger}(t)
$$

with time evolution

$$
\frac{d}{d t} U_{t}=-i\left(H_{S}+H_{F}+\lambda H_{I}\right) U_{t}
$$

where $H_{S} \in B\left(\mathbb{C}^{2}\right)$ is the Hamiltonian of the two-level atom, $H_{F} \in B(\mathscr{F} \otimes \mathscr{F})$ that of the field, and $\lambda H_{I} \in B\left(\mathbb{C}^{2}\right) \otimes B(\mathscr{F} \otimes \mathscr{F})$ is the interaction Hamiltonian. Define the interaction picture time evolution by 


$$
\hat{T}_{t *}(r):=U_{1}(t)^{\dagger} U_{2}(t)^{\dagger} T_{t *}(r) U_{2}(t) U_{1}(t),
$$

where $U_{1}(t):=e^{-i H_{S} t}$ and $U_{2}(t):=e^{-i H_{F} t}$ form the 'unperturbed' time evolution.

We now investigate $\hat{T}_{t}$ instead of $T_{t}$. Indeed, we are looking for a bound on the measurement infidelity $\delta=\sup _{S}\left\{\left\|T\left(\mathbf{1} \otimes \mathbf{1}_{S}(B)\right)-\mathbf{1}_{S}(A)\right\|\right\}$ of $T$. Yet if $\hat{B}:=U_{2}^{\dagger} B U_{2}$, then $\hat{T}\left(\mathbf{1}_{S}(\hat{B})\right)=T\left(\mathbf{1}_{S}(B)\right)$, so that

$$
\hat{\delta}=\sup _{S}\left\{\left\|\hat{T}\left(\mathbf{1} \otimes \mathbf{1}_{S}(\hat{B})\right)-\mathbf{1}_{S}(A)\right\|\right\}=\delta .
$$

If we find the interaction picture disturbance $\hat{\Delta}$, Corollary 1 will yield a bound on $\hat{\delta}$ and thus on $\delta$.

In the weak coupling limit $\lambda \downarrow 0, \hat{T}_{t}$ is given by

$$
\hat{T}_{t *}(\rho)=\hat{U}\left(t / \lambda^{2}\right)\left(\rho \otimes \phi_{\Omega} \otimes \phi_{0}\right) \hat{U}^{\dagger}\left(t / \lambda^{2}\right),
$$

where the evolution of the unitary cocycle $t \mapsto \hat{U}_{t}$ is described (see [1]) by a quantum stochastic differential equation or QSDE. Explicitly calculating the maximal added variances $\Sigma^{2}$ by solving the QSDE is in general rather nontrivial, if possible at all. (See [9] for the case of spontaneous decay, i.e. $\Omega=0$, with the map $\hat{T}_{t}$ restricted to the commutative algebra of homodyne measurement results.)

\subsubsection{Master equation for the open system}

Fortunately, in contrast to the somewhat complicated time evolution $\hat{T}_{t}$ of the combined system, the evolution restricted to the two-level system is both well known and uncomplicated. If we use $\lambda^{-2}$ as a unit of time, then the restricted evolution $\hat{R}_{t *}(r):=\operatorname{tr}_{\mathscr{F} \otimes \mathscr{F}} \hat{T}_{t *}(r)$ of the two-level system is known (see [3]) to satisfy the Master equation

$$
\frac{d}{d t} \hat{R}_{t *}(r)=L\left(\hat{R}_{t *}(r)\right),
$$

with the Liouvillian $L(r):=\frac{1}{2} i \Omega\left[e^{-i(\omega-E) t} V+e^{i(\omega-E) t} V^{\dagger}, r\right]-\frac{1}{2}\left\{V^{\dagger} V, r\right\}+V r V^{\dagger}$. In this expression, $E$ is the energy spacing of the two-level atom and $V^{\dagger}=\sigma_{+}, V=\sigma_{-}$ are its raising and lowering operators. In the case $\omega=E$ of resonance fluorescence, we obtain

$$
L(r)=\frac{1}{2} i \Omega\left[V+V^{\dagger}, r\right]-\frac{1}{2}\left\{V^{\dagger} V, r\right\}+V r V^{\dagger} .
$$

If we parametrize a state by its Bloch vector $\hat{R}_{t}^{*}(r)=\frac{1}{2}\left(\mathbf{1}+x \sigma_{x}+y \sigma_{y}+z \sigma_{z}\right)$, then Eq. (5) is simply the following differential equation on $\mathbb{R}^{3}$ :

$$
\frac{d}{d t}\left(\begin{array}{l}
x \\
y \\
z
\end{array}\right)=\left(\begin{array}{ccc}
-\frac{1}{2} & 0 & 0 \\
0 & -\frac{1}{2} & \Omega \\
0 & -\Omega & -1
\end{array}\right)\left(\begin{array}{l}
x \\
y \\
z
\end{array}\right)-\left(\begin{array}{l}
0 \\
0 \\
1
\end{array}\right) .
$$


This can be solved explicitly. For $\Omega \gg 1$, the solution approaches

$$
\left(\begin{array}{l}
x \\
y \\
z
\end{array}\right)=\left(\begin{array}{ccc}
e^{-\frac{1}{2} t} & 0 & 0 \\
0 & e^{-\frac{3}{4} t} \cos (\Omega t) & e^{-\frac{3}{4} t} \sin (\Omega t) \\
0 & -e^{-\frac{3}{4} t} \sin (\Omega t) & e^{-\frac{3}{4} t} \cos (\Omega t)
\end{array}\right)\left(\begin{array}{l}
x_{0} \\
y_{0} \\
z_{0}
\end{array}\right) .
$$

If we move to the interaction picture once more to counteract the Rabi oscillations, i.e. with $U_{1}(t)=e^{\frac{i}{2} \Omega t \sigma_{x}}$ and $U_{2}=\mathbf{1}$, we see that the time evolution is transformed to

$$
\left(\begin{array}{l}
x \\
y \\
z
\end{array}\right)=\left(\begin{array}{ccc}
e^{-\frac{1}{2} t} & 0 & 0 \\
0 & e^{-\frac{3}{4} t} & 0 \\
0 & 0 & e^{-\frac{3}{4} t}
\end{array}\right)\left(\begin{array}{l}
x_{0} \\
y_{0} \\
z_{0}
\end{array}\right)
$$

Since the trace distance $D(\rho, \tau)$ is exactly half the Euclidean distance between the Bloch vectors of $\rho$ and $\tau$, (see [21]), we see that $\Delta=\frac{1}{2}\left(1-e^{-3 t / 4}\right)$. For any measurement of $\sigma_{x}, \sigma_{y}$ or $\sigma_{z}$, we therefore have $\delta \geq \frac{1}{2}-\frac{1}{2} \sqrt{1-e^{-3 t / 2}}$ by Corollary 1 (remember that $t$ is in units of $\lambda^{-2}$ ).

\section{Collapse of the wave function}

The 'collapse of the wave function' may be seen as the flip side of the Heisenberg Principle. It states that if information is extracted from a system, then its states undergo a very specific kind of perturbation, called decoherence.

\subsection{Collapse for unbiased information transfer}

We start out by investigating unbiased information transfer. We prove a sharp upper bound on the amount of remaining coherence in terms of the measurement quality. Let $T: \mathscr{A} \otimes \mathscr{B} \rightarrow \mathscr{A}$ be a CP-map. Let $B \in \mathscr{B}$ be Hermitian, and define $A:=T(\mathbf{1} \otimes B)$.

Theorem 4 Suppose that $\psi_{x}$ and $\psi_{y}$ are eigenvectors of $A$ with different eigenvalues $x$ and $y$, respectively. Define $R: \mathscr{A} \rightarrow \mathscr{A}$ to be the restriction of $T$ to $\mathscr{A} \otimes \mathbf{1}$, and put $\Sigma^{2}:=\|(B, B)\|$. Then for all $\alpha, \beta \in \mathbb{C}$ with $|\alpha|^{2}+|\beta|^{2}=1$, we have

$$
\begin{aligned}
& D\left(R_{*}\left(\left|\alpha \psi_{x}+\beta \psi_{y}\right\rangle\left\langle\alpha \psi_{x}+\beta \psi_{y}\right|\right), R_{*}\left(|\alpha|^{2}\left|\psi_{x}\right\rangle\left\langle\left.\psi_{x}|+| \beta\right|^{2} \mid \psi_{y}\right\rangle\left\langle\psi_{y}\right|\right)\right) \\
& \quad \leq \frac{\Sigma /|x-y|}{\sqrt{1+4(\Sigma /|x-y|)^{2}}} .
\end{aligned}
$$

This bound is sharp in the sense that for all values of $\Sigma /|x-y|$, there exist $T, \psi_{x}$, $\psi_{y}, \alpha$ and $\beta$ for which (6) attains equality. 
Proof For sharpness of the bound, see Sect. 6.5. To prove (6), note that the 1.h.s. equals

$$
\sup \left\{\bar{\alpha} \beta\left\langle\psi_{x}, R(P) \psi_{y}\right\rangle+\text { c.c. } \mid P \in \mathscr{A}, P^{2}=P^{\dagger}=P\right\} .
$$

Furthermore, $2|\alpha||\beta| \leq 1$, so that it suffices to bound the 'coherence' $\left\langle\psi_{x}, R(P) \psi_{y}\right\rangle$ on all projections $P$. Now $(x-y)\left\langle\psi_{x}, R(P) \psi_{y}\right\rangle=\left\langle\psi_{x},[A, R(P)] \psi_{y}\right\rangle$, and $[A, R(P)]=$ $(P \otimes \mathbf{1}, \mathbf{1} \otimes B)-(\mathbf{1} \otimes B, P \otimes \mathbf{1})$. Thus,

$$
(x-y)\left\langle\psi_{x}, R(P) \psi_{y}\right\rangle=\left\langle\psi_{x},(P \otimes \mathbf{1}, \mathbf{1} \otimes B) \psi_{y}\right\rangle-\left\langle\psi_{x},(\mathbf{1} \otimes B, P \otimes \mathbf{1}) \psi_{y}\right\rangle,
$$

and we will bound these last two terms. In the notation of Lemma 1, we have $\Sigma=$ $\|g(\mathbf{1} \otimes B)\|$. Therefore,

$$
\begin{aligned}
\left\langle\psi_{x},(P \otimes \mathbf{1}, \mathbf{1} \otimes B) \psi_{y}\right\rangle & =\left\langle g(P \otimes \mathbf{1}) \psi_{x}, g(\mathbf{1} \otimes B) \psi_{y}\right\rangle \\
& \leq\left\|g(P \otimes \mathbf{1}) \psi_{x}\right\|\left\|g(\mathbf{1} \otimes B) \psi_{y}\right\| \\
& \leq \Sigma \sqrt{\left\langle\psi_{x},(P \otimes \mathbf{1}, P \otimes \mathbf{1}) \psi_{x}\right\rangle} .
\end{aligned}
$$

We will bound $\left\langle\psi_{x},\left(T\left(P^{2} \otimes \mathbf{1}\right)-T(P \otimes \mathbf{1})^{2}\right) \psi_{x}\right\rangle=\left\langle\psi_{x},\left(R(P)-R(P)^{2}\right) \psi_{x}\right\rangle$ in terms of the coherence. For brevity, denote $X_{x x^{\prime}}:=\left\langle\psi_{x}, X \psi_{x^{\prime}}\right\rangle$. Since $\psi_{x} \perp \psi_{y}$, we have

$$
\left(R(P)^{2}\right)_{x x} \geq\left|R(P)_{x x}\right|^{2}+\left|R(P)_{x y}\right|^{2},
$$

so that

$$
\left(R(P)-R(P)^{2}\right)_{x x} \leq R(P)_{x x}\left(1-R(P)_{x x}\right)-\left|R(P)_{x y}\right|^{2} .
$$

Since $x(1-x) \leq \frac{1}{4}$ for all $x \in \mathbb{R}$, this is at most $\frac{1}{4}-\left|R(P)_{x y}\right|^{2}$. All in all, we have obtained

$$
(P \otimes \mathbf{1}, \mathbf{1} \otimes B)_{x y} \leq \Sigma \sqrt{\frac{1}{4}-\left|R(P)_{x y}\right|^{2}}
$$

and of course the same for $x \leftrightarrow y$. Plugging these into Eq. (7) yields

$$
|x-y|\left|R(P)_{x y}\right| \leq 2 \Sigma \sqrt{\frac{1}{4}-\left|R(P)_{x y}\right|^{2}}
$$

or equivalently $\left|R(P)_{x y}\right| \leq \frac{\Sigma /|x-y|}{\sqrt{1+4(\Sigma /|x-y|)^{2}}}$, which was to be proven.

Consider the ideal case of perfect $(\Sigma=0)$ information transfer. Suppose that the system $\mathscr{A}$ is initially in the coherent state $\left|\alpha \psi_{x}+\beta \psi_{y}\right\rangle\left\langle\alpha \psi_{x}+\beta \psi_{y}\right|$. Then, Theorem 4 says that, after the information transfer to the ancilla $\mathscr{B}$, the system $\mathscr{A}$ cannot be distinguished from one that started out in the 'incoherent' state

$$
|\alpha|^{2}\left|\psi_{x}\right\rangle\left\langle\left.\psi_{x}|+| \beta\right|^{2} \mid \psi_{y}\right\rangle\left\langle\psi_{y}\right|
$$


Fig. 5 Bound on the coherence as a function of $\Sigma /|x-y|$. All points above this curve are forbidden; all points below are allowed

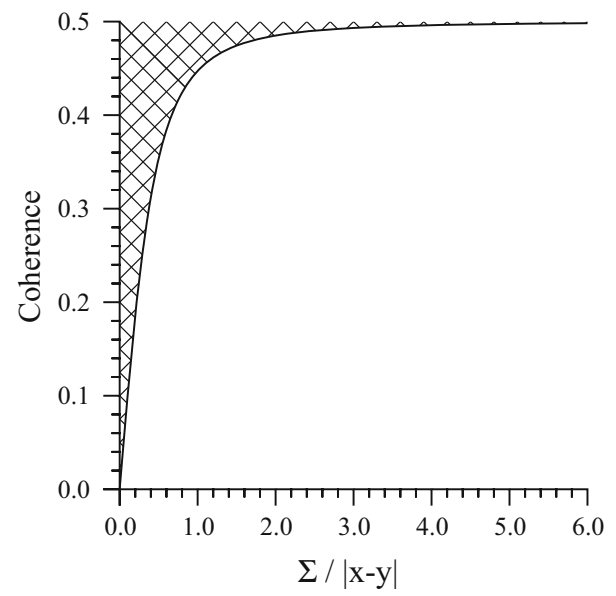

As far as the behaviour of $\mathscr{A}$ is concerned, it is therefore completely harmless to assume that a collapse

$$
\left|\alpha \psi_{x}+\beta \psi_{y}\right\rangle\left\langle\alpha \psi_{x}+\left.\beta \psi_{y}|\mapsto| \alpha\right|^{2} \mid \psi_{x}\right\rangle\left\langle\left.\psi_{x}|+| \beta\right|^{2} \mid \psi_{y}\right\rangle\left\langle\psi_{y}\right|
$$

has occurred at the start of the procedure.

Now consider the other extreme of a measurement which leaves all states intact, i.e. $R^{*}(\rho)=\rho$ for all $\rho$. Then there exist states for which the 1.h.s. of Eq. (6) equals $\frac{1}{2}$, forcing $\Sigma \rightarrow \infty$; no information can be obtained. This is Werner's formulation of the Heisenberg Principle [31].

Theorem 4 thus unifies the Heisenberg Principle and the collapse of the wave function. For $\Sigma=0$, we have a full decoherence, whereas if all states are left intact, we have $\Sigma \rightarrow \infty$. For all intermediate cases, the bound (6) on the remaining coherence is an increasing function of $\Sigma /|x-y|$ (Fig. 5).

This agrees with physical intuition: decoherence between $\psi_{x}$ and $\psi_{y}$ is expected to occur in case the information transfer is able to distinguish between the two. This is the case if the variance is small w.r.t. the differences in mean.

\subsection{Application: Perfect qubit measurement}

In Sect. 2, we have encountered the von Neumann Qubit measurement. Now consider any perfect measurement $T$ of $\sigma_{z}$ with pointer $\mathbf{1} \otimes\left(\delta_{+}-\delta_{-}\right)$which leaves $|\uparrow\rangle\langle\uparrow|$ and $|\downarrow\rangle\langle\downarrow|$ in place, i.e. $R_{*}(|\uparrow\rangle\langle\uparrow|)=|\uparrow\rangle\langle\uparrow|$ and $R_{*}(|\downarrow\rangle\langle\downarrow|)=|\downarrow\rangle\langle\downarrow|$. (Such a measurement is called nondestructive.) Theorem 4 then reads

$$
R^{*}(|\alpha \uparrow+\beta \downarrow\rangle\langle\alpha \uparrow+\beta \downarrow|)=|\alpha|^{2}|\uparrow\rangle\left\langle\left.\uparrow|+| \beta\right|^{2} \mid \downarrow\right\rangle\langle\downarrow|,
$$

illustrated in Fig. 6. 
Fig. 6 Collapse on the Bloch sphere for perfect measurement

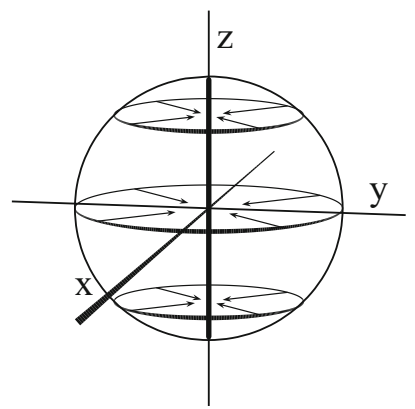

Incidentally, the trace distance between the centre of the Bloch sphere and its surface is $\frac{1}{2}$, so that we read off $\Delta=\sup \left\{D\left(R^{*}(\rho), \rho\right) ; \rho \in \mathscr{S}\left(M_{2}\right)\right\}=\frac{1}{2}$. This was predicted by Theorem 3 .

\subsection{Collapse of the wave function for general measurement}

We will prove a sharp bound on the remaining coherence in general information transfer. For technical convenience, we will focus attention on nondestructive measurements. A measurement of $A$ is called 'nondestructive' (or 'conserving' or 'quantum nondemolition') if it leaves the eigenstates of $A$ intact, so that repetition of the measurement will yield the same result. For example, the measurement in Sect. 2.2 is nondestructive, the one in Sect. 5.3 is destructive. Restriction to nondestructive measurements is quite common in quantum measurement theory (see [23]).

Let $T: B(\mathscr{H}) \otimes \mathscr{B} \rightarrow B(\mathscr{H})$ be a CP-map, let $A \in B(\mathscr{H})$ and $B \in \mathscr{B}$ be Hermitean and suppose that $\left\{\psi_{i}\right\}$ is an orthogonal basis of eigenvectors of $A$, with eigenvalues $a_{i}$. Define the measurement infidelity $\delta:=\sup _{S}\left\{\| T\left(\mathbf{1} \otimes \mathbf{1}_{S}(B)\right)-\right.$ $\left.\mathbf{1}_{S}(A) \|\right\}$.

Corollary 2 Suppose that $T$ is nondestructive, i.e. $R_{*}\left(\left|\psi_{i}\right\rangle\left\langle\psi_{i}\right|\right)=\left|\psi_{i}\right\rangle\left\langle\psi_{i}\right|$ for all $\psi_{i}$, with $R$ the restriction of $T$ to $B(\mathscr{H}) \otimes \mathbf{1}$. Then if $\delta \in\left[0, \frac{1}{2}\right]$, and $a_{i} \neq a_{j}$,

$$
\begin{gathered}
D\left(R_{*}\left(\left|\alpha \psi_{i}+\beta \psi_{j}\right\rangle\left\langle\alpha \psi_{i}+\beta \psi_{j}\right|\right),\left(|\alpha|^{2}\left|\psi_{i}\right\rangle\left\langle\left.\psi_{i}|+| \beta\right|^{2} \mid \psi_{j}\right\rangle\left\langle\psi_{j}\right|\right)\right) \\
\leq \sqrt{\delta(1-\delta)}
\end{gathered}
$$

This bound is sharp in the sense that for all $\delta \in\left[0, \frac{1}{2}\right]$, there exist $T, \psi_{i}, \psi_{j}, \alpha$ and $\beta$ for which (8) attains equality.

Proof For sharpness, see Sect. 6.5. Choose a set $S$ such that $a_{i} \in S$ and $a_{j} \notin S . T$ is an unbiased measurement of $T\left(\mathbf{1} \otimes \mathbf{1}_{S}(B)\right)$ with pointer $\mathbf{1}_{S}(B)$ and maximal added variance $\Sigma^{2} \leq \delta(1-\delta)$ (cf. the proof of Corollary 1 ). We will prove that $\psi_{i}$ and $\psi_{j}$ are eigenvectors of $T\left(\mathbf{1} \otimes \mathbf{1}_{S}(B)\right)$ with eigenvalues $x$ and $y$ which differ at least $1-2 \delta$.

Let $P_{i}:=\left|\psi_{i}\right\rangle\left\langle\psi_{i}\right|$. Since $T$ is nondestructive, we have $\left\langle\psi_{j}, R\left(P_{i}\right) \psi_{j}\right\rangle=$ $\left\langle\psi_{j}, P_{i} \psi_{j}\right\rangle$ for all $j$. Apparently, $R\left(P_{i}\right)$ has only one nonzero diagonal element, a 1 at 
Fig. 7 Bound on the coherence in terms of $\delta$. All points above the curve are forbidden; all points below are allowed

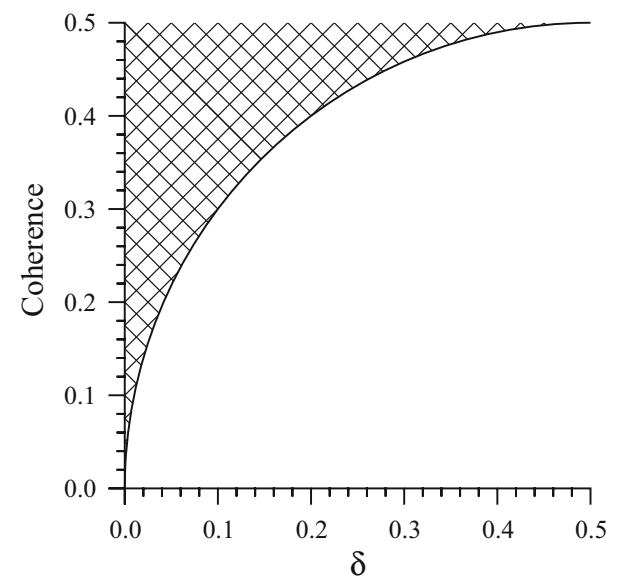

position $(i, i)$. Since $R\left(P_{i}\right) \geq 0$, this implies $R\left(P_{i}\right)=P_{i}$. Then $\left(P_{i} \otimes \mathbf{1}, P_{i} \otimes \mathbf{1}\right)=0$, so that by Cauchy-Schwarz $\left(P_{i} \otimes \mathbf{1}, \mathbf{1} \otimes \mathbf{1}_{S}(B)\right)=0$. Since $P_{i}=T\left(P_{i} \otimes \mathbf{1}\right)$, we have

$$
\left[T\left(\mathbf{1} \otimes \mathbf{1}_{S}(B)\right), P_{i}\right]=\left(P_{i} \otimes \mathbf{1}, \mathbf{1} \otimes \mathbf{1}_{S}(B)\right)-\left(\mathbf{1} \otimes \mathbf{1}_{S}(B), P_{i} \otimes \mathbf{1}\right)=0 .
$$

Therefore, $\psi_{i}$ is an eigenvector of $T\left(\mathbf{1} \otimes \mathbf{1}_{S}(B)\right)$, with eigenvalue $x$, say. By a similar reasoning, $\psi_{j}$ is also an eigenvector, denote its eigenvalue by $y$.

Since $\left\|T\left(\mathbf{1} \otimes \mathbf{1}_{S}(B)\right)-\mathbf{1}_{S}(A)\right\| \leq \delta$, we have in particular

$$
\begin{aligned}
\left\|\left(T\left(\mathbf{1} \otimes \mathbf{1}_{S}(B)\right)-\mathbf{1}_{S}(A)\right) \psi_{i}\right\| & =|x-1| \leq \delta, \\
\left\|\left(T\left(\mathbf{1} \otimes \mathbf{1}_{S}(B)\right)-\mathbf{1}_{S}(A)\right) \psi_{j}\right\| & =|y| \leq \delta,
\end{aligned}
$$

so that $|x-y| \geq 1-2 \delta$. We can now apply Theorem 4. On the 1.h.s. of the bound (6), we may substitute

$$
R_{*}\left(|\alpha|^{2}\left|\psi_{i}\right\rangle\left\langle\left.\psi_{i}|+| \beta\right|^{2} \mid \psi_{j}\right\rangle\left\langle\psi_{j}\right|\right)=|\alpha|^{2}\left|\psi_{i}\right\rangle\left\langle\left.\psi_{i}|+| \beta\right|^{2} \mid \psi_{j}\right\rangle\left\langle\psi_{j}\right|
$$

on account of $T$ being nondestructive. On the r.h.s., we substitute $\Sigma=\sqrt{\delta(1-\delta)}$ and $|x-y|=(1-2 \delta)$. Strikingly enough, this yields the bound

$$
(\sqrt{\delta(1-\delta)} /(1-2 \delta)) / \sqrt{1+4\left(\delta(1-\delta) /(1-2 \delta)^{2}\right)}=\sqrt{\delta(1-\delta)} .
$$

For perfect measurement $(\delta=0)$, this yields

$$
R_{*}\left(\left|\alpha \psi_{i}+\beta \psi_{j}\right\rangle\left\langle\alpha \psi_{i}+\beta \psi_{j}\right|\right)=|\alpha|^{2}\left|\psi_{i}\right\rangle\left\langle\left.\psi_{i}|+| \beta\right|^{2} \mid \psi_{j}\right\rangle\left\langle\psi_{j}\right|,
$$

all coherence between $\psi_{i}$ and $\psi_{j}$ must vanish. This collapse of the wave function is illustrated in the lower left corner of Fig. 7. On the other hand, if all states are left 
intact so that $R_{*}=\mathrm{id}$, then we must have $\delta=\frac{1}{2}$; no information can be gained. This is illustrated in the upper right corner of Fig. 7. Corollary 2 is a unified description of the Heisenberg Principle and the collapse of the wave function.

\subsection{Application: Nondestructive qubit measurement}

In quantum information theory, a $\sigma_{z}$-measurement is often taken to yield output +1 or -1 , according to whether the input was $|\uparrow\rangle$ or $|\downarrow\rangle$. It is nondestructive if it leaves the states $|\uparrow\rangle$ and $|\downarrow\rangle$ intact, yet it is only unbiased if it is perfect. Corollary 2 shows that in the nondestructive case, the Bloch sphere collapses to the cigar-shaped region depicted in Fig. 8.

Current single-qubit read-out technology is now in the regime where the bound (8) becomes significant; in [16], a nondestructive measurement of a SQUID-qubit was described, with experimentally determined measurement infidelity $\delta=0.13$. The bound then equals 0.336 .

\subsection{Sharpness of the bounds}

We have yet to prove sharpness of all bounds. Let

$$
V_{+}:=\left(\begin{array}{cc}
\sqrt{1-p} & 0 \\
0 & \sqrt{p}
\end{array}\right) \quad, \quad V_{-}:=\left(\begin{array}{cc}
\sqrt{p} & 0 \\
0 & \sqrt{1-p}
\end{array}\right)
$$

and define $T: M_{2} \otimes \mathscr{C}(\Omega) \rightarrow M_{2}$ by $T(X \otimes f):=f(+1) V_{+} X V_{+}+f(-1) V_{-} X V_{-}$. For $p=0$, this is the von Neumann measurement. As a measurement of $\sigma_{z}$ with pointer $B:=\left(\delta_{+}-\delta_{-}\right) /(1-2 p)$, we have $\delta=p$. This yields bounds on the disturbance and on the coherence. Corollary 1 and Theorem 3 yield $\Delta \geq \frac{1}{2}-\sqrt{p(1-p)}$, corollary 2 and Theorem 4 yield

$$
D\left(R_{*}(|\alpha \uparrow+\beta \downarrow\rangle\langle\alpha \uparrow+\beta \downarrow|),\left(|\alpha|^{2}|\uparrow\rangle\left\langle\left.\uparrow|+| \beta\right|^{2} \mid \downarrow\right\rangle\langle\downarrow|\right)\right) \leq \sqrt{p(1-p)} .
$$

Fig. 8 Collapse on the Bloch sphere with $\delta=0.01$

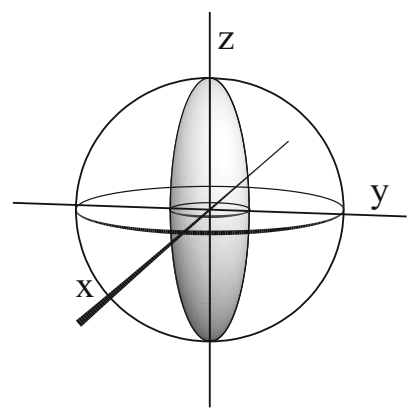


We now explicitly calculate the restriction of $T$ to $M_{2}$ and find

$$
R_{*}(r)=\left(\begin{array}{cc}
r_{11} & 2 \sqrt{p(1-p)} r_{12} \\
2 \sqrt{p(1-p)} r_{21} & r_{22}
\end{array}\right) .
$$

The maximal remaining coherence occurs for $\alpha=\beta=1 / \sqrt{2}$, for which it equals $\sqrt{p(1-p)}$. The maximal disturbance equals $\Delta=\frac{1}{2}-\sqrt{p(1-p)}$. This shows all bounds to be sharp.

\section{Conclusion}

Our investigation of joint measurement, the Heisenberg Principle and decoherence has yielded the following results.

(I) Theorem 2 provides a sharp, state-independent bound on the performance of unbiased joint measurement of noncommuting observables. In the case of perfect ( $\Sigma=0$ ) measurement of one observable, it implies that no information whatsoever $\left(\Sigma^{\prime}=\infty\right)$ can be gained on the other.

(II) Theorem 3 (for unbiased information transfer) and Corollary 1 (for general information transfer) provide a sharp, state-independent bound on the performance of a measurement in terms of the maximal disturbance that it causes. In the case of zero disturbance, when all states are left intact, it follows that no information can be obtained. This is the Heisenberg Principle.

(III) Theorem 4 (for unbiased information transfer) and Corollary 2 (for general information transfer) provide a sharp upper bound on the amount of coherence which can survive information transfer. For perfect information transfer, all coherence vanishes. This clearly proves that decoherence on a system is a mathematical consequence of information transfer out of this system. If, on the other hand, all states are left intact, then it follows that no information can be obtained. This is the Heisenberg Principle. Theorem 4 and Corollary 2 connect these two extremes in a continuous fashion; they form a unified description of the Heisenberg Principle and the collapse of the wave function.

Acknowledgements Open access funding provided by Max Planck Society. I would like to thank Hans Maassen for his invaluable guidance and advice.

Open Access This article is distributed under the terms of the Creative Commons Attribution 4.0 International License (http://creativecommons.org/licenses/by/4.0/), which permits unrestricted use, distribution, and reproduction in any medium, provided you give appropriate credit to the original author(s) and the source, provide a link to the Creative Commons license, and indicate if changes were made.

\section{References}

1. Accardi, L., Frigerio, A., Lu, Y.G.: The weak coupling limit as a quantum functional central limit. Commun. Math. Phys. 131, 537-570 (1990)

2. Arthurs, E., Kelly, J.: On simultaneous measurement on a pair of conjugate observables. Bell Syst. Tech. J. 44, 725 (1965)

3. Bouten, L., Guţă, M., Maassen, H.: Stochastic Schrödinger equations. J. Phys. A 37, 3189-3209 (2004) 
4. Hall, M.: Prior information: how to circumvent the standard joint-measurement uncertainty relation. Phys. Rev. A 69, 052113 (2004)

5. Heisenberg, W.: Über den anschaulichen Inhalt der quantentheoretischen Kinematik und Mechanik. Z. Phys. 43, 172-198 (1927)

6. Hepp, K.: Quantum theory of measurement and macroscopic observables. Helv. Phys. Acta 45, 237248 (1972)

7. Holevo, A.S.: Probabilistic and Statistical Aspects of Quantum Theory. North Holland Publishing Company, Amsterdam (1982)

8. Ishikawa, S.: Uncertainty relations in simultaneous measurements for arbitrary observables. Rep. Math. Phys. 29, 257-273 (1991)

9. Janssens, B., Bouten, L.: Optimal pointers for joint measurement of $\sigma_{x}$ and $\sigma_{z}$ via homodyne detection. J. Phys. A 39, 2773-2790 (2006)

10. Janssens, B., Maassen, H.: Information transfer implies state collapse. J. Phys. A: Math. Gen. 39, 9845-9860 (2006)

11. Joos, E., Zeh, H.: The emergence of classical properties through interaction with the environment. Z. Phys. B 59, 223-243 (1985)

12. Kadison, R.V.: A generalized Schwarz inequality and algebraic invariants for operator algebras. Ann. Math. 2(56), 494-503 (1952)

13. Kadison, R.V., Ringrose, J.R.: Fundamentals of the Theory of Operator Algebras, Volume II, Advanced Theory. Academic Press, Cambridge (1986)

14. Kennard, E.: Zur Quantenmechanik einfacher Bewegungstypen. Z. Phys. 44, 326-325 (1927)

15. Lahti, P.: Coexistence and joint measurability in quantum mechanics. Int. J. Theor. Phys. 42(5), 893906 (2003)

16. Lupaşcu, A.E.A.: High-contrast dispersive readout of a superconducting flux qubit using a nonlinear resonator. Phys. Rev. Lett. 96, 127003 (2006)

17. Majgier, K., Maassen, H., Życzkowski, K.: Protected subspaces in quantum information. Quantum Inf. Process. 9(3), 343-367 (2010)

18. Miyadera, T., Imai, H.: Heisenberg's uncertainty principle for simultaneous measurement of positive operator-valued measures. Phys. Rev. A 78, 052119 (2008)

19. Miyadera, T.: Relation between strength of interaction and accuracy of measurement for a quantum measurement. Phys. Rev. A 83, 052119 (2011)

20. Miyadera, T., Loveridge, L., Busch, P.: Approximating relational observables by absolute quantities: a quantum accuracy-size trade-off. J. Phys. A: Math. Theor. 49, 185301 (2016)

21. Nielsen, M., Chuang, I.: Quantum Computation and Quantum Information. Cambridge University Press, Cambridge (2000)

22. Ozawa, M.: Universally valid reformulation of the Heisenberg uncertainty principle on noise and disturbance in measurement. Phys. Rev. A 67, 042105 (2003)

23. Peres, A.: Quantum Theory: Concepts and Methods. Kluwer Academic Publishers, Dordrecht (1993)

24. Polterovich, L.: Quantum unsharpness and symplectic rigidity. Lett. Math. Phys. 102(3), 245-264 (2012)

25. Polterovich, L.: Symplectic geometry of quantum noise. Commun. Math. Phys. 327(2), 481-519(2014)

26. Robertson, H.: The uncertainty principle. Phys. Rev. 34, 163-164 (1929)

27. Sewell, G.: On the mathematical structure of quantum measurement theory. Rep. Math. Phys. 56, 271-290 (2005)

28. Takesaki, M.: Theory of Operator Algebras I. Springer, New York (1979)

29. von Neumann, J.: Mathematische Grundlagen der Quantenmechanik. Springer, Berlin (1932)

30. Werner, R.: Optimal Cloning of Pure States. Phys. Rev. A 58, 1827-1832 (1998)

31. Werner, R.: Quantum information theory-an invitation. Quantum Inf. 173, 14-57 (2001)

32. Zurek, W.: Environment-Induced Superselection Rules. Phys. Rev. D 26, 1862-1880 (1982) 